\title{
Spinal cord stimulation for chronic pain: the importance of early referral
}
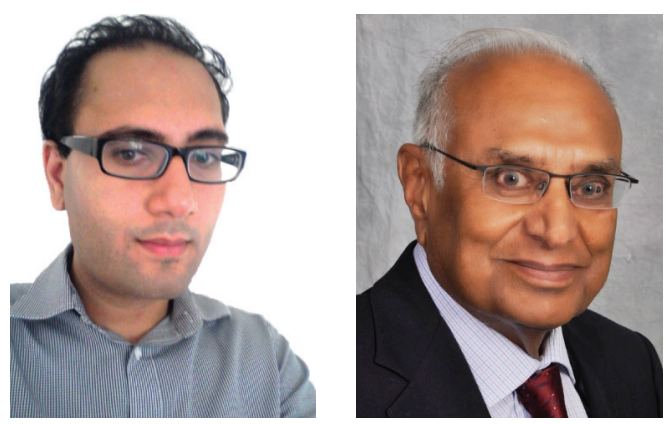

Syed Rizvi $i^{*, 1}$ \& Krishna Kumar ${ }^{\dagger, 2}$

Chronic pain is a disease unto itself, responsible for significant physical and psychosocial suffering. The prevalence of neuropathic pain has been estimated at about 8 to $9 \%$ in the USA [1]. It responds poorly to standard pharmacological and surgical therapies. For these patients spinal cord stimulation (SCS) is the only viable treatment option. The use of SCS is supported by randomized controlled trials, meta-analyses, and costeffectiveness data. Its strengths lie in its reversibility, programmability, low risk and specificity. Its benefits are evidenced by improved pain relief, functional status, health-related quality of life, and reduced demand for healthcare resources [2-5].

Initially conceived of as a therapy of last resort, SCS has gradually acquired first-line status for the treatment of chronic neuropathic pain. There is widespread agreement amongst experts that patients presenting with neuropathic pain who do not respond to conventional treatments by 12 to 16 weeks should be offered a trial of SCS. We have demonstrated that efficacy of SCS treatment is time dependent with success rates exceeding $80 \%$ if implantation occurs within 2 years of symptom onset, compared with
$15 \%$ for patients whose implants happened 20 years after the onset of pain [6-9].

Our data reveals that present mean wait times of 5.45 years roughly translate into a long-term success rate of $47 \%[3,6]$. A sub-analysis indicated that patients face significant delays in accessing SCS at each step of the treatment continuum. Patients made initial contact with a family physician an average of 3.4 months after developing a pain syndrome. Family physicians managed patients for 11.9 months. These barriers are influenced by the limited time spent identifying the cause of pain and the lack of expertise in treating chronic pain. In healthcare systems where payment is largely fee-for-service, a quick patient turnover is financially advantageous, leading to a disincentive to critically orient pain care. This is particularly concerning because chronic pain can neither be diagnosed nor managed in a hurried fashion [6].

The most significant point of delay occurred at the level of specialist care. Specialists then took over management for an additional 39.8 months on average. Neurosurgeons were quickest to make a referral, whereas, non-implanting

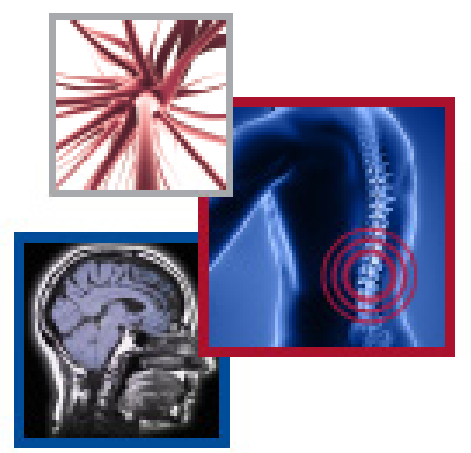

\author{
KEYWORDS \\ - chronic pain • complex \\ regional pain syndrome \\ - failed back surgery \\ syndrome $\bullet$ health care \\ process improvement $\bullet$ \\ neuropathic pain $\bullet$ spinal cord \\ simulation • spine intervention \\ - wait times
}

\footnotetext{
'Department of Neurology, University of Saskatchewan, Saskatoon SK, Canada 
anesthetists were most likely to delay implantation. In fact, referral for SCS treatment took 2.15 years longer if a non-implanting anesthetist versus a neurosurgeon received the initial patient referral.

Neurologists and neurosurgeons tended to consider SCS earlier, within 3 years of patient care. By contrast, non-implanting anesthetists and orthopedic surgeons managed cases for a mean of 4.84 and 4.30 years, respectively. We postulate that this discrepancy may reflect differing practice patterns, skill sets, and knowledge levels concerning neuromodulation. It is conceivable, for instance, that orthopedic surgeons may be more amenable to consider lumbosacral spine surgery, such as spinal fusion with or without instrumentation. The expected corollary is that 1 year may elapse before the success or failure of surgery is observable, further delaying institution of SCS. Similarly, anesthetists may initially prefer a variety of less invasive pharmacological treatments or nerve blocks to transient, eventually waning benefit, before contemplating neuromodulation upon failure of these procedures [6]. It remains a sad reality, that even in the face of overwhelming evidence to the contrary, the false dogma of SCS as the last-ditch recourse continues to linger in professional circles.

Timely access to effective treatment is a major concern for healthcare systems [8-10]. Unfortunately, patients cycle through ineffective treatments despite recommendations by various pain societies that SCS should be considered early. The International Association for the Study of Pain suggests that wait times for assessment by a pain specialist should not exceed 8 weeks $[9,10]$. If these recommendations are adopted, wait times for SCS will automatically fall. The key to optimizing success of SCS is to employ strict patient selection criteria and to offer SCS earlier. These findings should motivate policymakers and healthcare bodies to develop strategies that shorten wait times. Barriers to SCS include lack of uptake and awareness among healthcare providers, patients and payers; ongoing reimbursement concerns; and fragmentation of pain-care delivery. Early SCS is likely to enhance functional restoration, resulting in improved return to work rates with significant implications for generalized acceptance of this therapy [4-7].

Despite its robust evidence base, real-world SCS utilization remains disappointingly low. A retrospective analysis of 16,455 patients with failed back surgery syndrome (FBSS) disclosed that only $2.4 \%$ of eligible patients underwent SCS while $97.6 \%$ received re-operation [13]. It is all too common for otherwise ideal candidates for SCS to be shunted through recurrent cycle(s) of back surgery to dwindling benefit and detrimental outcomes. Typically, 14\% of those with chronic back pain have had back surgery, with $19 \%$ receiving re-operation $[6,11]$.

Challenges on the professional and reimbursement fronts are compounded by the low public profile carried by SCS. According to a recent European poll survey, $61 \%$ of respondents who stood to benefit from SCS were unaware of its existence [11]. Moreover, it appeared that $87 \%$ of patients had trialed four or more treatment options before SCS was ever considered. Among those aware of SCS, few had been informed by their doctor or specialist, with most relying on self-discovery through television or internet [11,12].

When access to SCS is curtailed, patients suffer, a point accentuated by functional neuroimaging studies which reveals that gray matter atrophy is accelerated to a loss of $1.3 \mathrm{~cm}^{3}$ annually or 5 to $11 \%$ of volume per year in patients with chronic pain. The magnitude of this decrease is equivalent to the gray matter volume lost in 10 to 20 years of normal aging [14,15]. This suggests that chronic pain behaves like a neurodegenerative disorder and strengthens our contention that early implantation is a necessary consideration.

Even as the burden of chronic pain continues to grow, research for pain is hardly a priority. An analysis of NIH funding patterns revealed that funding for pain research actually declined by an average of $9.4 \%$ per year between 2003 and 2007 . The percent of the total NIH budget allocated to pain research fell to a meager $0.61 \%$ in 2007 . This critical research gap must be addressed as current therapies fail to prevent disease progression or even induce sustained remissions [16]. In the meantime, we hope that SCS will continue advancing to earlier and more prominent stages in the treatment for chronic pain.

In conclusion, early SCS for neuropathic pain disorders bolsters success rates, improves functional endpoints such as return to employment, and may hopefully prevent the dreaded cerebral atrophy which has been shown to occur over time.

\footnotetext{
Acknowlegements

Krishna Kumar passed away on 23 April 2014 at the age 83. Well-known for his research in the treatment of chronic pain through spinal cord stimulation, Dr. Kumar built a distinguished career as an innovative neurosurgeon,
} 
pioneering neuromodulator, and tireless advocate for patients living with chronic pain. Dr Kumar was a prolific academic and earned numerous accolades including the Order of Canada, the Queen's Diamond Jubilee Medal and the International Neuromodulation Society's Giant of Neuromodulation Award in 2011 for his work. As lead author of the seminal PROCESS trial, Dr. Kumar helped spinal cord stimulation gain the legitimacy it needed in both the scientific and public arenas and facilitated its transformation into an accepted, foundational therapy for chronic neuropathic pain. Empathetic and exceedingly kind, elegant and eloquent, Dr Kumar was never one to slow down, and was the quintessential gentleman. It has been a distinct privilege of mine to have enjoyed the brilliance of Dr. Kumar, his life and his work, for the past decade. Knowing Krishna has enriched my life and lives of many:
"Every day you have to learn something. It is up to you, if you want to be ahead in the pack, the middle of the pack or behind in the race. You hold the key to your success. I want to be ahead in the race and I don't care how far the next guy is behind me." - (K Kumar)

\section{Financial \& competing interests disclosure}

K Kumar was a consultant for Medtronic Inc. and Boston Scientific and received fees and grants for consulting, being a steering committee member, and research funding for past projects. S Rizvi has no conflict of interest. The authors have no other relevant affliations or financial involvement with any organization or entity with a financial interest in or financial conflict with the subject matter or materials discussed in the manuscript apart from those disclosed. No writing assistance was utilized in the production of this manuscript.

\section{References}

1 Yawn BP, Wollan PC, Weingarten TN, Watson JC, Hooten WM, Melton LJ 3rd. The prevalence of neuropathic pain: clinical evaluation compared with screening tools in a community population. Pain Med. 10 (3), 586-593 (2009).

2 Kumar K, Rizvi S. Historical and present state of neuromodulation in chronic pain. Curr. Pain Headache Rep. 18(1), 387 (2014).

3 Kumar K, Taylor RS, Jacques L et al. The effects of spinal cord stimulation in neuropathic pain are sustained: a 24-month follow-up of the prospective randomized controlled multicenter trial of the effectiveness of spinal cord stimulation. Neurosurgery 63(4) 762-770 (2008).

4 Kumar K, Rizvi S. Cost-effectiveness of spinal cord stimulation therapy in management of chronic pain. Pain Med. 14(11), 1631-1649 (2013).

5 Kumar K, Hunter G, Demeria D. Spinal cord stimulation in treatment of chronic benign pain: challenges in treatment planning and present status, a 22-year experience. Neurosurgery. 58(3), 481-496 (2006).
6 Kumar K, Rizvi S, Nguyen R et al. Impact of wait-times on spinal cord stimulation therapy outcomes. Pain Pract. (2013) (In press).

7 Kumar K, Rizvi S, Bishop S. Spinal cord stimulation is effective in management of complex regional pain syndrome (CRPS) I:

Fact or Fiction. Neurosurgery 69(3), 566-580 (2011).

8 Stanton-Hicks MD, Burton AW, Bruehl SP et al. An updated interdisciplinary clinical pathway for CRPS: report of an expert panel. Pain Pract. 2(1), 1-16 (2002).

9 International Association for the Study of Pain 2010 Annual Report. International Association for the Study of Pain (2010). www.iasppain.org/files/Content/ ContentFolders/AboutIASP/ IASPAnnualReport_2010.pdf

10 Lynch ME, Campbell F, Clark AJ et al. A systematic review of the effect of waiting for treatment for chronic pain. Pain. 136(1-2), 97-116 (2008).

11 IML Research. The painful truth survey: State of pain management in Europe. www.epresspack.net/mnr/ download $/$ id $=4763 \& \mathrm{pn}=937949-\mathrm{pdf}$
12 Thomson S, Jacques L. Demographic characteristics of patients with severe neuropathic pain secondary to failed back surgery syndrome. Pain Pract. 9(3), 206-215 (2009).

13 Choi J, Babu R, Ranjith MS et al. 157 Utilization of Spinal Cord Stimulation in Patients with Failed Back Surgery Syndrome [abstract]. Neurosurgery. 71, E562-E563 (2012).

14 Apkarian AV, Sosa Y, Sonty $S$ et al. Chronic back pain is associated with decreased prefrontal and thalamic gray matter density. J. Neurosci. 24(46), 10410-10415 (2004).

15 Apkarian AV, Scholz J. Shared mechanisms between chronic pain and neurodegenerative disease. Drug Discov. Today Dis. Mech. 3 , 319-326 (2006).

16 Bradshaw DH, Empy C, Davis P, Lipschitz D, Nakamura Y, Chapman CR. Trends in funding for research on pain: a report on the National Institutes of Health grant awards over the years 2003 to 2007. J. Pain 9(12), 1077-1087 (2008). 